\title{
CLINICAL TIPS FOR YOUR FIRST PROCEDURES
}

\section{By Kieran Nandhra \& Calvin Kund}

W e have all been there and we shall always have that initial fear. Doing things for the first time on an actual patient is always nerve-racking, whether you are an experienced dentist, trying out a new technique, or a junior student taking your very first steps onto clinic. However, we have gathered some tips through our own experiences to make you feel more comfortable and at ease during any procedure you haven't done before as detailed below and in Figure 1.

\section{Preparation: practice beforehand}

Reducing nerves starts with your ability to prepare before the patient gets in. Although it is not always the same, practising in your simulation lab or speaking to a colleague, who has undergone the same experience can help with guidance and highlight areas you need to focus on. If you are practising a clinical technique in preparation for a patient, such as root canal therapy or a crown, it is always best to seek feedback from a tutor. Finding out where you can improve or highlighting your struggles to colleagues and tutors may help you on the day as you'll know how to overcome these challenges.

\section{Preparation: anticipate challenges}

Whenever we face something new it creates the fear of the unknown. Anticipating challenges and things that can go wrong beforehand can help with management. For example, with root canal therapy, one of the things that can go wrong is the unfortunate case of a hypochlorite accident. By acknowledging this and then revising or recapping on how to manage such situations you may feel more confident on the day. More often than not, procedures are completed without huge complications, although there are a few challenges to overcome. Being prepared is the best way to feel less overwhelmed, should the event of a complication arise.

\section{Preparation: Be organised}

Psychologically, being organised helps with your treatment on the day. Arriving early onto clinic and setting everything up means that you aren't stressing at the time during treatment. It also means that your time management is better and, most importantly, gives a reassuring impression to your patients. We believe that laying out all your instruments on the work bench in the correct order can help with efficiency. Following this, it can help, to quickly go through in your head all the steps you are going to take so that when the patient steps through the door you are ready to go. Alternatively, bringing in notes for complex cases (e.g. crown reduction guides) has proven handy in our experiences.

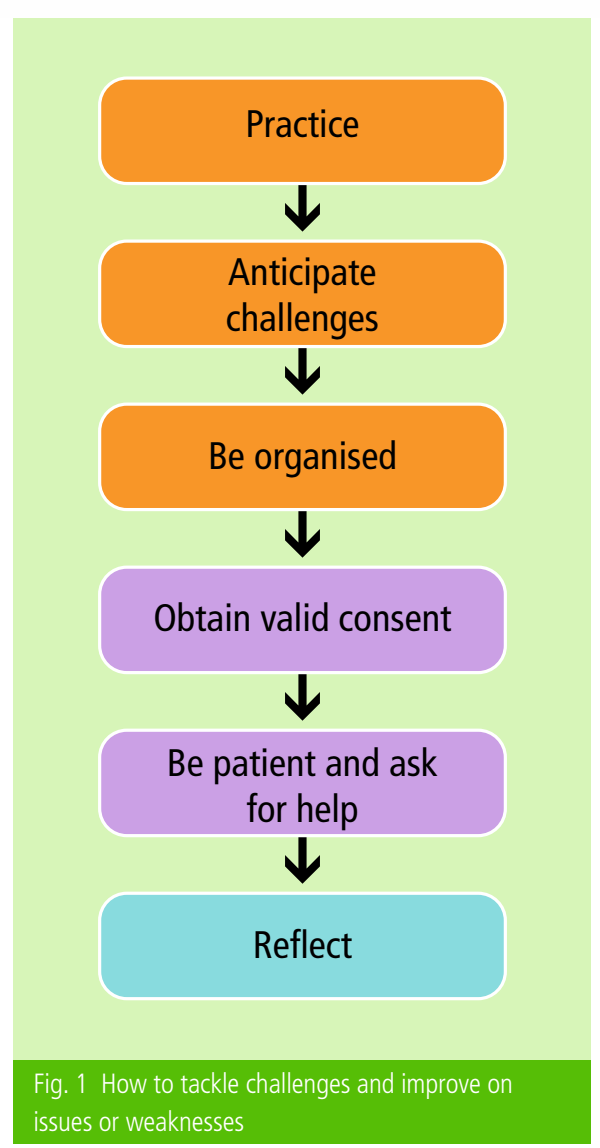

During Treatment: Informed consent GDC principle three states that you need to, 'Obtain valid consent.' This includes providing all the risks and benefits. By informing the patient of the complications and communicating effectively you can put forward some potential concerns and challenges that you anticipated. For example, every root canal therapy treatment option has a chance of failing. If this is not highlighted to the patient and the complication arises it complicates matters and puts you at risk for further issues.

\section{During treatment: Be patient and ask for help if necessary}

There is no need to rush procedures that you have never done before. Applying a time constraint on yourself only seems to distract your focus on the task in hand. In addition, when you take more time you tend to be more careful, rushing may cause you to make undue mistakes and you may need to deal with a harder situation than in the first place. Moreover, if you are really struggling there is no harm in asking for help. If anything, it is more professional to ask for help when you are struggling than to forge ahead, acting as though you are fine. While it may feel good to finish earlier, remember being in clinics with many clinicians around is a unique opportunity to further your learning. GDC principle seven is a good reference to make as it outlines, 'Maintain, develop and work within our professional knowledge and skills, therefore it is one our duties to understand when we can carry out treatment safely and understand when we need help.

\section{After treatment: Reflect}

Reflecting on what went well, what could have been better and putting into motion an action plan to help improve confidence and competence aids you to feel less nervous should you need to carry out the procedure again. Remember that as a student it is fine to feel inundated. Furthermore, you should readily ask your patients, tutors and nurses on improvements. As time progresses you will work your way to becoming a competent clinician. Practising dentistry is a challenging feat, it is a steady learning curve but can be rewarding. In order to feel less concerned about stepping out of the comfort zone you need to be prepared and feel stress-free on the day.

Kieran Nandhra \& Calvin Kund 